\title{
DROIT ET POLITIQUE DES DÉCHETS INDUSTRIELS ANALYSE COMPARATIVE DE SIX STRATÉGIES NATIONALES EN EUROPE
}

Maître Stanley Chaney, Maître Christine Baudoin

CL\&A

Cette analyse comparative de six stratégies nationales (Allemagne, Belgique, Espagne, France, Italie, Luxembourg) de droit et politique des déchets industriels dans des pays de l'Union Européenne est le fruit d'une collaboration de plusieurs intervenants du secteur de l'environnement :

- CL\&A (Cournot Lemétais \& Associés), cabinet d'avocats d'affaires appelé à intervenir régulièrement dans le domaine du droit de l'environnement, membre du réseau international Pannone Law Group, en collaboration avec Monsieur José Santamaria du cabinet Bufete Pinto Ruiz, et Maître Maude Vézina, du Cabinet CL\&A.

- Tredi : leader français du traitement des déchets industriels spéciaux difficiles.

This comparative analysis of six national strategies (Germany, Belgium, Spain, France, Italy, Luxembourg) <law and polities of industrial waste> in the countries of the European Union, has the benefit of the colloboration of many specialists in the environmental sector:

-CL\&A (Cournot, Lemétais \& Associés) Business lawyers practice regulary called to intervene in the province of environmental law, a member of Pannone Law Group - an international network, in collaboration with Mr José Santamaria of the Bufete Pinto Ruiz practice and Maître Maude Vézina of CL\&A,

-Tredi : French leader of environmental treatment of special industrial waste difficulties.

\section{TEXTES FONDATEURS : NATIONS DÉFRICHEUSES/NATIONS SUIVEUSES}

Si chaque pays étudié dispose d'une législation déchets, on peut distinguer :

- Allemagne, Belgique et France dans une approche défricheuse au cours des années 70 ;

- Luxembourg, Espagne et Italie dans une approche suiveuse au cours des années 80 .

Entre ces deux étapes, les textes européens fondateurs ont été votés au Parlement (cf. par exemple définition de la notion de déchet) et ont permis l'impulsion de la deuxième vague des pays suiveurs.

L'exemple type est la législation déchets italienne qui peut se résumer quasi exclusivement à une transcription en droit national des directives européennes (cf. aussi l'exemple caricatural de la Grèce sur ce thème).

\section{DÉFINITIONS DE LA NOTION DE DÉCHETS}

La définition de la notion de déchets est à la base même de toute approche en matière de droit et politique déchets industriels.

Nombre des pays européens ont adopté la définition communautaire qui est également celle retranscrite dans les conventions internationales : substance ou objet « dont le détenteur veut se défaire ou se débarrasser ou dont il a l'intention de se défaire en vertu des dispositions en vigueur $"$.

Cette définition tente une synthèse entre critères objectifs (obligations) et critères subjectifs (intentions d'abandon) et peut, à cet égard, constituer un territoire privilégié pour des acteurs procéduriers (exemple transformation de terre de déblai en terre de remblai).

\begin{tabular}{|c|c|c|}
\hline \multicolumn{3}{|c|}{$\begin{array}{l}\text { Tableau I : Textes fondateurs par pays } \\
\text { et année de parution }\end{array}$} \\
\hline Pays & $\begin{array}{l}\text { Texte fondateur } \\
\text { d'une politique déchets industriels }\end{array}$ & $\begin{array}{l}\text { Année } \\
\text { de parution }\end{array}$ \\
\hline Allemagne & $\begin{array}{l}\text { «Waste Disposal Act » } \\
\text { (Abfallbeseitigungsgesetz) (AbfG) }\end{array}$ & 1972 \\
\hline Belgique & $\begin{array}{l}\text { Loi du } 22 \text { juillet } 1974 \\
\text { sur les déchets toxiques }\end{array}$ & 1974 \\
\hline Espagne & $\begin{array}{l}\text { Loi cadre sur les résidus dangereux } \\
\mathrm{n}^{\circ} 20 \text { du } 14 \text { mai } 1986\end{array}$ & 1986 \\
\hline France & $\begin{array}{l}\text { Loi } n^{\circ} 75-633 \text { relative à l'élimination des } \\
\text { déchets et à la récupération des matériaux }\end{array}$ & 1975 \\
\hline Italie & $\begin{array}{l}\text { Décret du Président de la République } \\
\mathrm{n}^{\circ} 915 \text { du } 10 \text { septembre } 1982 \text { (DPR 915/82) }\end{array}$ & 1982 \\
\hline Luxembourg & $\begin{array}{l}\text { Loi du } 26 \text { juin } 1980 \text { réglementant la détention } \\
\text { et l'élimination, le ramassage, le transport } \\
\text { et de manière générale toute activité } \\
\text { ayant trait aux déchets }\end{array}$ & 1980 \\
\hline
\end{tabular}


Tableau 2 : Définition de la notion de déchets dans les différents pays étudiés

\begin{tabular}{|c|c|c|c|}
\hline Pays & Définition générique & $\begin{array}{l}\text { Classification } \\
\text { générale }\end{array}$ & $\begin{array}{l}\text { Textes de loi } \\
\text { de référence }\end{array}$ \\
\hline Allemagne & $\begin{array}{l}\text { Deux manières de définir le concept « déchet » : sur la base de critères subjectifs et } \\
\text { fondés sur l'intention du propriétaire : «tout bien mobile dont le propriétaire veut se } \\
\text { débarrasser », sur une base objective (c'est-à-dire selon les caractéristiques physiques) : } \\
\text { "tout résidu dont l'élimination appropriée est nécessaire, dans l'intérêt de la santé publique } \\
\text { et particulièrement pour la protection de l'environnement ». }\end{array}$ & & $\begin{array}{l}\text { Waste Disposal } \\
\text { Act. } 1972 \\
1986 \text { Waste Act. }\end{array}$ \\
\hline $\begin{array}{l}\text { Belgique } \\
\text { - Région } \\
\text { flamande }\end{array}$ & $\begin{array}{l}\text { Trois définitions légales du concept « déchets 》: } \\
\text { - Déchets : «toute substance ou tout objet dont le détenteur veut se débarrasser, } \\
\text { se débarrasse ou est tenu de se débarrasser en vertu du présent décret ou en vertu } \\
\text { des dispositions prises en application de ce décret ». } \\
\text { - Déchets dangereux : « déchets qui présentent ou peuvent présenter un danger } \\
\text { particulier pour la santé de l'homme ou pour l'environnement ou qui doivent être } \\
\text { traités, neutralisés ou détruits dans des établissements spéciaux ». }\end{array}$ & $\begin{array}{l}\text { Déchets } \\
\text { Déchets dangereux }\end{array}$ & $\begin{array}{l}\text { Décret du } 2 / 07 / 81 \\
\text { concernant la } \\
\text { gestion des déchets } \\
\text { Décret du } 5 / 07 / 85 \\
\text { relatif aux déchets } \\
\text { spéciaux }\end{array}$ \\
\hline $\begin{array}{l}\text { - Région } \\
\text { wallone }\end{array}$ & $\begin{array}{l}\text { "Toute substance ou tous objets qui relèvent des catégories figurant en annexe I dont } \\
\text { le détenteur se défait ou dont il a l'intention ou l'obligation de se défaire ». }\end{array}$ & $\begin{array}{l}\text { Déchets ménagers, agri- } \\
\text { coles, industriels, dange- } \\
\text { reux (hors toxiques) }\end{array}$ & $\begin{array}{l}\text { Ordonnance du } \\
7 / 03 / 9 \mid \text { relative à la } \\
\text { prévention et à la }\end{array}$ \\
\hline $\begin{array}{l}\text { - Région } \\
\text { bruxelloise }\end{array}$ & $\begin{array}{l}\text { "Toute substance ou tout objet qui relèvent des catégories figurant en annexe I dont } \\
\text { le détenteur se défait ou dont il a l'intention ou l'obligation de se défaire ». }\end{array}$ & $\begin{array}{l}\text { Déchets ménagers, } \\
\text { particuliers, dangereux }\end{array}$ & gestion des déchets \\
\hline Espagne & $\begin{array}{l}\text { "Résidu »: Toute substance ou tout objet appartenant à l'une quelconque des catégorie } \\
\text { figurant en annexe à cette loi dont son possesseur se départit ou a l'intention ou l'obli- } \\
\text { gation de se départir. En tout cas seront considérés comme tels les objets figurant dans } \\
\text { le Catalogue Européen de Résidus (CER) approuvé par les institutions communautaires. } \\
\text { "Résidus urbains ou municipaux » : Ceux générés dans les résidences particulières, } \\
\text { commerces, bureaux et services, ainsi que tous ceux qui n'ont pas la qualification de } \\
\text { dangereux et qui par leur nature ou leur composition peuvent être assimilés à ceux } \\
\text { produits dans les activités antérieures. Seront également qualifiés de résidus urbains } \\
\text { ceux énumérés ci-après : résidus provenant du nettoyage des voies publiques, espaces } \\
\text { verts, espaces récréatifs et plages; cadavres d'animaux domestiques, ainsi que meubles, } \\
\text { outils et véhicules abandonnés; résidus et déchets provenant de travaux mineurs } \\
\text { de construction et de réparation d'habitation. } \\
\text { "Résidus dangereux »: Ceux qui figurent sur la liste des résidus dangereux approuvée } \\
\text { dans le Décret Royal } 952 / 1997 \text { (RCL I997, I729), ainsi que les récipients et contenants } \\
\text { qui les auraient contenus; ceux qui sont qualifiés comme dangereux par la norme com- } \\
\text { munautaire et ceux qui pourront l'être par le gouvernement en conformité avec ce qui } \\
\text { est précisé dans la norme européenne et dans les accords internationaux auxquels } \\
\text { l'Espagne est partie. }\end{array}$ & $\begin{array}{l}\text { Résidus industriels et } \\
\text { industriels spéciaux } \\
\text { (norme régionale) } \\
\text { Résidus sanitaires }\end{array}$ & $\begin{array}{l}\text { Loi } n^{\circ} 10 / 1998 \text { du } \\
21 \text { avril } 1998 \text { sur les } \\
\text { produits dangereux } \\
\text { (BOE } n^{\circ} 96 \\
22 \text { avril } 1998 \text { ) }\end{array}$ \\
\hline France & $\begin{array}{l}\text { Déchets : « tout résidu d'un processus de production, de transformation ou d'utilisation, toute } \\
\text { substance, matériau, produit ou plus généralement tout bien, meuble, abandonné ou que } \\
\text { son détenteur destine à l'abandon ». } \\
\text { Déchets retenus pour réglementation : ceux « de nature à produire des effets nocifs sur } \\
\text { le sol, la flore et la faune, à dégrader les sites ou les paysages, à polluer l'air ou les eaux, } \\
\text { à engendrer des bruits ou des odeurs et d'une façon générale, à porter atteinte à la santé } \\
\text { de l'homme et à l'environnement ». }\end{array}$ & $\begin{array}{l}\text { Déchets ménagers } \\
\text { et assimilés } \\
\text { Déchets industriels }\end{array}$ & $\begin{array}{l}\text { Loi du } 15 / 07 / 75 \\
\text { modifiée }\end{array}$ \\
\hline Italie & $\begin{array}{l}\text { Déchets : « toute substance ou objet qui entre dans la catégorie reportée à l'annexe I et } \\
\text { dont le détenteur se débarrasse ou a décidé ou a l'obligation de s'en défaire ». } \\
\text { Déchets urbains : } \\
\text { - déchets domestiques ou encombrants provenant de locaux ou de lieux d'habitation, } \\
\text { - déchets provenant du balayage des rues, } \\
\text { - déchets de toute nature ou provenant des rues et/ou espaces privés se trouvant } \\
\text { dans les lieux publics tels que rue, place, plage, rive, } \\
\text { - déchets non dangereux provenant d'un usage autre que l'habitation mais qui sont } \\
\text { assimilés aux déchets urbains en raison de leur nature et de la qualité et de la quantité } \\
\text { aux termes de l'article } 2 \text { I (2G), } \\
\text { - déchets provenant des espaces verts (jardins, parcs). } \\
\text { - déchets provenant d'exhumation. } \\
\text { Déchets spéciaux: } \\
\text { - déchets provenant de l'activité agricole et agro-alimentaire, } \\
\text { - déchets provenant de l'activité de démolition et de construction } \\
\text { - déchets dangereux provenant de l'activité de fouilles, } \\
\text { - déchets provenant d'activité industrielle, arsenal, commerciale et de services, } \\
\text { - déchets dérivant de l'activité de récupération et de traitement des déchets, } \\
\text { - boue produite par le traitement des eaux, } \\
\text { - épuration des eaux, fumées, } \\
\text { - déchets dérivés d'activité hospitalière, } \\
\text { - machines et appareils détériorés, véhicules à moteur, remorques, pièces de rechange. } \\
\text { Déchets dangereux : tous autres déchets listés à l'annexe } D \text {. }\end{array}$ & $\begin{array}{l}\text { Déchets toxiques } \\
\text { et nocifs } \\
\text { - Autres déchets: } \\
\text { radioactifs, déchets de } \\
\text { mines et de carrières, } \\
\text { déchets agricoles }\end{array}$ & $\begin{array}{l}\text { Décret législatif du } \\
5 \text { février } 1997 n^{\circ} 22 \\
\text { sur les déchets } \\
\text { industriels }\end{array}$ \\
\hline
\end{tabular}


Tableau 2: Définition de la notion de déchets dans les différents pays étudiés (suite)

\begin{tabular}{|c|c|c|c|}
\hline Pays & Définition générique & $\begin{array}{l}\text { Classification } \\
\text { générale }\end{array}$ & $\begin{array}{l}\text { Textes de loi } \\
\text { de référence }\end{array}$ \\
\hline Luxembourg & $\begin{array}{l}\text { Déchets : « toute substance ou tout objet qui relève des catégories figurant à l'annexe I de } \\
\text { la présente loi et d'une manière générale, tout bien meuble abandonné ou que son détenteur } \\
\text { destine à l'abandon ou dont il a l'obligation de se défaire ». Sont considérés comme des } \\
\text { déchets au sens de la présente loi les produits et substances destinés à la valorisation } \\
\text { jusqu'à ce que ces produits ou substances, ainsi que les matières premières secondaires } \\
\text { ou l'énergie qui résultent de l'opération de valorisation soient réintroduits dans } \\
\text { le circuit économique. } \\
\text { Déchets dangereux : «les déchets qui sont caractérisés par leur nature ou l'activité qui les a } \\
\text { produits ou qui sont rendus dangereux par leurs constituants ou leurs propriétés ». }\end{array}$ & $\begin{array}{l}\text { Déchets } \\
\text { Déchets toxiques } \\
\text { et dangereux } \\
\text { Huiles } \\
\text { Autres déchets : } \\
\text { - déchets provenant } \\
\text { de l'industrie du dioxyde } \\
\text { de titane, } \\
\text { - déchets nucléaires, } \\
\text { - déchets particuliers } \\
\text { (déchets d'animaux) }\end{array}$ & $\begin{array}{l}\text { Loi du } 17 \text { juin } 1994 \\
\text { relative à } \\
\text { la prévention et à } \\
\text { la gestion des } \\
\text { déchets et modifiant } \\
\text { la loi du } 26 \text { juin } \\
1980\end{array}$ \\
\hline
\end{tabular}

En France, la jurisprudence considère comme déchet toute matière usée non encore traitée et ce, même si son détenteur la destine au commerce et non à l'abandon.

Aujourd'hui encore, cette définition même du déchet est au cœur des litiges relatifs aux transferts de déchets transfrontaliers (cf. l'affaire "Wastec-Strobel » du nom de cette société de Sarrebruck qui se bat pour faire reconnaître son droit à importer chez les agriculteurs lorrains des boues d'épuration du Bade-Wurternberg ou de Rhénanie Palatinat, en attente d'un arbitrage européen après condamnation par le TGI de Metz).

La définition du déchet est précisée dans chacun des six pays retenus dans cette étude par l'existence de nomenclatures nationales visant à répertorier les différentes catégories de déchets.

Cette démarche, fortement inspirée de l'Annexe I de la directive 75/442/CEE modifiée et du catalogue européen des déchets, vise d'abord à discriminer déchets ménagers et déchets industriels.

Chaque pays a ensuite redéfini, à l'intérieur de la famille déchets industriels, un certain nombre de catégories spécifiques : déchets d'emballage, déchets hospitaliers, huiles usagers, $\mathrm{PCB} / \mathrm{PCT}$, déchets radioactifs.

\section{HIÉRARCHISATION DES OBJECTIFS POLITIQUES}

Aucun des pays étudiés n'échappe à la déclaration d'intention et, si chaque nation partage une vision commune du tiercé gagnant (réduction à la source, recyclage ou réutilisation des déchets, élimination des déchets dans le respect de l'environnement général), la traduction sur le terrain diffère fortement selon les pays.

On mesure cet écart entre une nation défricheuse comme l'Allemagne qui passe d'une « loi sur le stockage des déchets » en 1972 à une « loi sur la prévention et la suppression des déchets ॥ en 1986, tandis que l'Espagne (nation suiveuse) pose à peine en 1988 le principe de la création d'une infrastructure nécessaire au traitement et à la réduction des résidus (plan national de résidus industriels toxiques et dangereux).

Néanmoins, avec l'entrée en vigueur de l'acte unique en 1987 et plus récemment du traité de Maastricht
( ${ }^{\text {er }}$ novembre 1993), les politiques environnement en général (et déchets en particulier) des nations européennes visent à une évidente harmonisation.

Citons pour exemple la politique globale belge en matière d'environnement qui est basée sur le principe énoncé dans l'article I30R CEE : «les exigences en matière d'environnement doivent être intégrées dans la définition et la mise en œuvre des autres politiques de la Communauté », conformément au traité de l'Union Européenne et aux directives et règlements d'application qui le complètent (principe du « pollueur payeur », prévention et classification des déchets en fonction de leur dangerosité et système d'autorisation).

\section{MODALITÉS PRATIQUES DES FILIĖRES ET MISE EN CEUVRE DES OBJECTIFS POLITIQUES}

Trait commun à tous les pays étudiés, la pression réglementaire s'exerce dans un ordre inverse à celui défini dans le cadre de la hiérarchisation des objectifs politiques.

Ainsi quel que soit le pays ciblé, l'élimination des déchets industriels y est fortement réglementée.

L'industriel a la responsabilité de l'élimination de ses déchets selon des filières internes ou externes soumises obligatoirement à l'autorité administrative.

L'Allemagne (tout comme la France avec la déclaration et l'autorisation) présente deux régimes de réglementation selon la complexité de l'unité :

- la procédure de détermination de projet,

- la procédure simplifiée d'autorisation.

La Belgique soumet (tout comme la France) l'instruction des demandes d'autorisation à enquête publique et à notice préalable d'incidences sur l'environnement ou à études d'incidences.

Par ailleurs, tant en région wallonne qu'en région BruxellesCapitale, le législateur systématise actuellement la délivrance de permis uniques unifiant les diverses autorisations antérieures.

De plus, les approches fédérales de la Belgique et l'Espagne s'inscrivent dans une logique d'autorisation délivrée par l'organisme régional compétent avec surveillance ultérieure organisée par les autorités locales et fédérées. En Belgique, la nouvelle Constitution coordonnée du 17 février 1994 a 


\begin{tabular}{|c|c|c|}
\hline \multicolumn{3}{|c|}{$\begin{array}{l}\text { Tableau } 3 \text { : Principes de base des stratégies } \\
\text { nationales en matière de déchets industriels }\end{array}$} \\
\hline Pays & $\begin{array}{l}\text { Instruments de } \\
\text { la stratégie nationale } \\
\text { en matière de déchets } \\
\text { industriels }\end{array}$ & Principes de base \\
\hline Allemagne & $\begin{array}{l}\text { Loi Déchets } 1986 \text { « Loi sur } \\
\text { la prévention et la suppres- } \\
\text { sion de déchets » }\end{array}$ & $\begin{array}{l}\text { Philosophie des } 3 \mathrm{~V} \text { : préven- } \\
\text { tion (Vermeidung), réduction } \\
\text { (Verminderung), récupération } \\
\text { (Verwertung) }\end{array}$ \\
\hline Belgique & $\begin{array}{l}\text { Loi du 22/07/74 sur les } \\
\text { déchets toxiques, Loi } \\
\text { spéciale du } 16 / 07 / 93 \text { visant } \\
\text { à la structure fédérale } \\
\text { de l'État }\end{array}$ & $\begin{array}{l}\text { - Principe du pollueur-payeur } \\
\text { (article I3OR CEE) } \\
\text { - Prévention et contrôle } \\
\text { - Mesures à caractère fiscal }\end{array}$ \\
\hline Espagne & $\begin{array}{l}\text { Plan national de résidus } \\
\text { industriels toxiques et } \\
\text { dangereux (1988) }\end{array}$ & $\begin{array}{l}\text { - Création de l'infrastructure } \\
\text { nécessaire au traitement et à } \\
\text { la réduction des résidus, } \\
\text { - Coordination de la gestion } \\
\text { des résidus }\end{array}$ \\
\hline France & $\begin{array}{l}\text { Loi du I5/07/75 modifiée } \\
\text { (et directive du I8/03/9I) }\end{array}$ & $\begin{array}{l}\text { - Principe de prévention ou de } \\
\text { réduction/valorisation } \\
\text { - Principe de transparence des } \\
\text { circuits }\end{array}$ \\
\hline Italie & Loi $n^{\circ} 44$ I du 29/09/87 & $\begin{array}{l}\text { - Respecter les principes de } \\
\text { base relatifs à l'exercice d'une } \\
\text { activité liée à l'intérêt public } \\
\text { - Encourager les filières } \\
\text { recyclage, récupération }\end{array}$ \\
\hline Luxembourg & Loi du 28 juin 1980 & $\begin{array}{l}\text { - Assurer un traitement et un } \\
\text { stockage sans nuisance } \\
\text { - Promouvoir le tri, le } \\
\text { recyclage et le compostage } \\
\text { - Contrôle et } \\
\text { professionnalisation des } \\
\text { filières }\end{array}$ \\
\hline
\end{tabular}

transféré la quasi totalité des compétences déchets aux trois autorités fédérées (régions wallonne, flamande et bruxelloise) : l'autorité fédérale ne conservant plus que trois compétences explicites (norme des produits, protection contre les radiations ionisantes et transits de déchets).

L'Italie dispose d'un régime réglementaire avec délivrance d'une autorisation régionale pour l'établissement d'élimination.

Au Luxembourg, la loi du 27 juillet 1993 a créé une décharge nationale pour les déchets non ménagers.

Par ailleurs, tout établissement d'élimination des déchets est classé établissement dangereux dont l'existence et l'exploitation sont soumises d'une part à l'existence d'autorisation en vertu de la loi sur la gestion des déchets et d'autre part à une autorisation conformément à la loi sur les établissements classés.

En France, les notions de Predi et de déchets ultimes sont venues compléter le cahier des charges du producteur de déchets industriels qui devait déjà, soit bénéficier d'un agrément pour procéder à l'élimination, soit recourir à une société agréée.

A l'inverse de la filière élimination, les filières relatives au recyclage des déchets et plus encore à la réduction à la source, ne sont que peu ou très rarement encadrées par des contraintes réglementaires.

L'Allemagne avec tant son « dual system » que son décret emballage, a fait en la matière figure de précurseur.

Le décret emballage visait, pour la première fois en Europe, à rendre producteurs et distributeurs financièrement et pratiquement responsables du devenir des emballages de leur production.

Cette expérience a également constitué avec la contribution financière volontaire au "dual system ॥ la première dérive d'un système d'incitation : l'industriel préférant le paiement d'une taxe supplémentaire à la gestion interne de ses emballages.

Le succès du «point vert » auprès du grand public a accentué ce phénomène de dérive en revalorisant l'emballage comme support marketing, au lieu d'aider à sa minimisation.

La loi déchets de 1986 a, par ailleurs, inscrit le recyclage au titre des préoccupations de l'industrie, introduisant ainsi le principe de définition de taux de recyclage par matériau (verre, étain...) négociés entre le ministère de l'Environnement et l'Industrie.

Le législateur a également introduit en Belgique le principe d'une éco-taxe susceptible de frapper à l'unité toute sorte d'objets « de courte durée de vie » (récipients pour boissons non consignés, appareils jetables, piles électriques non rechargeables...).

En France aussi, une politique de gestion des emballages industriels a été récemment mise en œuvre. Cependant, à ce jour, tous les décrets concernant la valorisation des produits recyclés, prévus par la loi de 1975 n'ont pas encore été adoptés, confirmant bien la hiérarchisation des priorités donnée précédemment.

II semble néanmoins que les techniques contractuelles (Eco-emballages, Adelphe, Cyclamed) puissent bénéficier du soutien des pouvoirs publics.

Bien qu'au Luxembourg la législation impose le principe de récupération préalable des déchets avant élimination, ce qui aliéna l'élaboration d'un projet d'installation en vue de recueillir les déchets industriels, il n'existe pas à l'heure actuelle de décharge. En effet, l'unique projet a dû être abandonné suite à une décision du Tribunal Administratif en décembre 1998.

Le Luxembourg n'a donc d'autre ressource que de contacter les pays voisins pour transférer ses déchets.

Par ailleurs, il existe une difficulté au Luxembourg de s'équiper d'infrastructures lourdes et coûteuses et ce, eu égard à une production insuffisante sur son territoire.

\section{RESPONSABILITÉ CIVILE ET RESPONSABILITÉ PÉNALE}

Dans chacune des législations nationales des pays étudiés, la responsabilité en matière de déchets peut s'exercer aux plans pénal ou civil.

Mais pour chacune des législations étudiées, la notion de responsabilité recouvre d'abord et bien souvent uniquement 
Tableau 4 : Responsabilité civile et pénale dans les différents pays étudiés

\begin{tabular}{|c|c|c|}
\hline Pays & Responsabilité civile & Responsabilité pénale \\
\hline Allemagne & $\begin{array}{l}\text { Loi de responsabilité } \\
\text { environnementale 1991 } \\
\text { Code Civil (Art. 823, } 906 \\
\text { et 1004) }\end{array}$ & $\begin{array}{l}\text { Code Pénal (Art. } 324 \text { à } \\
\text { 330) (en relation avec } \\
\text { la loi déchets 1986) }\end{array}$ \\
\hline Belgique & $\begin{array}{l}\text { Code Civil (Art. I } 382 \text { à I384) } \\
\text { Loi du } 22 \text { juillet I } 974 \text { (Art. 7) } \\
\text { (Responsabilité objective du } \\
\text { producteur de déchets toxiques) }\end{array}$ & $\begin{array}{l}\text { Code Pénal } \\
\text { (en relation avec la loi } \\
\text { du } 22 \text { juillet } 1974 \text { sur } \\
\text { les déchets toxiques) }\end{array}$ \\
\hline Espagne & BOE n 96,22 avril 1998 & $\begin{array}{l}\text { BOE } n^{\circ} 96 \\
22 \text { avril } 1998\end{array}$ \\
\hline France & $\begin{array}{l}\text { Code Civil (Art. I } 382 \text { à I386) } \\
\text { (Responsabilité « prolongée » } \\
\text { du producteur) }\end{array}$ & $\begin{array}{l}\text { Nouveau Code Pénal } \\
\text { en application de la loi } \\
\text { du } 16 \text { décembre } 1992 \\
\text { (en relation avec la loi } \\
\text { du I } 5 \text { juillet } 1975 \text { sur } \\
\text { les déchets) }\end{array}$ \\
\hline Italie & $\begin{array}{l}\text { Code Civil (notamment au titre } \\
\text { de la responsabilité civile) }\end{array}$ & $\begin{array}{l}\text { Code Pénal } \\
\text { (Art. } 590,635 \text { et } 639 \\
\text { au titre des dommages } \\
\text { sur la personne et } \\
\text { sur la propriété) }\end{array}$ \\
\hline Luxembourg & Code Civil (Art. I 382 et I384) & $\begin{array}{l}\text { Code Pénal (en relation } \\
\text { avec la loi } \\
\text { du } 26 \text { juin 1980) }\end{array}$ \\
\hline
\end{tabular}

les dommages causés à un tiers ou à la propriété et non à l'environnement en tant que tel (à l'inverse du système américain).

C'est la raison pour laquelle, en particulier en France, des prérogatives spécifiques ont récemment été accordées à certaines associations agréées de protection de l'environnement (cf. sur ce point l'approche voisine belge au titre de la loi du 12 janvier 1993, concernant un droit d'action en matière d'environnement, attribué au ministère public et aux associations de défense de l'environnement).

Toujours concernant la France, il convient de rappeler que la réforme du Code Pénal (effective au ${ }^{\mathrm{er}}$ mars 1994) prévoit, et notamment en matière de législation déchets, une responsabilité pénale des personnes morales en sus de celle des personnes physiques qui les dirigent.

Les réflexions proches de l'Allemagne, la Belgique et la France autour des notions respectivement de « stricte responsabilité » (sans preuve de la faute à pondérer selon le système complexe de la « cause présumée »), « responsabilité objective » (fondée sur le support des conséquences par celui qui a créé le risque), « responsabilité prolongée » (non transférable à un tiers en cas de remise du déchet) sont autant d'apports aux schémas actuels moins aboutis de nations comme l'Italie et l'Espagne se référant classiquement, en la matière, encore aux règles de droit commun (le Luxembourg étant notamment, avec l'absence de fourniture d'une faute déterminée, dans un schéma intermédiaire). II convient enfin d'évoquer la responsabilité administrative à travers deux exemples pris en France et en Belgique.

En Belgique, cette compétence a été établie par le vote d'une loi en date du 29 juillet 1991 sur la motivation formelle des actes administratifs qui contraint toutes les administrations à motiver d'une manière formelle et adéquate leur décision.

En France, l'administration peut être poursuivie en carence et jugée responsable en cas d'abstention à agir lorsque la loi lui en fait obligation. L'arrêt Francovitch est susceptible d'entraîner la mise en œuvre de la responsabilité de l'administration jusque dans le défaut de transposition d'une directive communautaire.

Maître Stanley Chaney, Maître Christine Baudoin, CL\&A - 5, rue Beaujon - 75008 Paris

\section{A LA RECHERCHE DU VÊTEMENT ÉCOLOGIOUE}

\author{
Gérard Bertolini, \\ économiste, directeur de recherche au CNRS
}

Pierre Melquiot,

Docteur ingénieur en génie industriel et environnement, responsable de projets au Cetih (Centre technique des industries de ('habillement),

Informer et éclairer le consommateur ainsi que les industriels concernés; sensibiliser à l'écologique; verdir les productions et les achats (promouvoir à la fois l'offre et la demande), faire de l'écologie un argument de vente...

Format $16 * 21$ - 164 pages - $1999-180 \mathrm{~F}$ TTC franco de port (170,62 F HT - TVA 5,5\%:9,38 F)

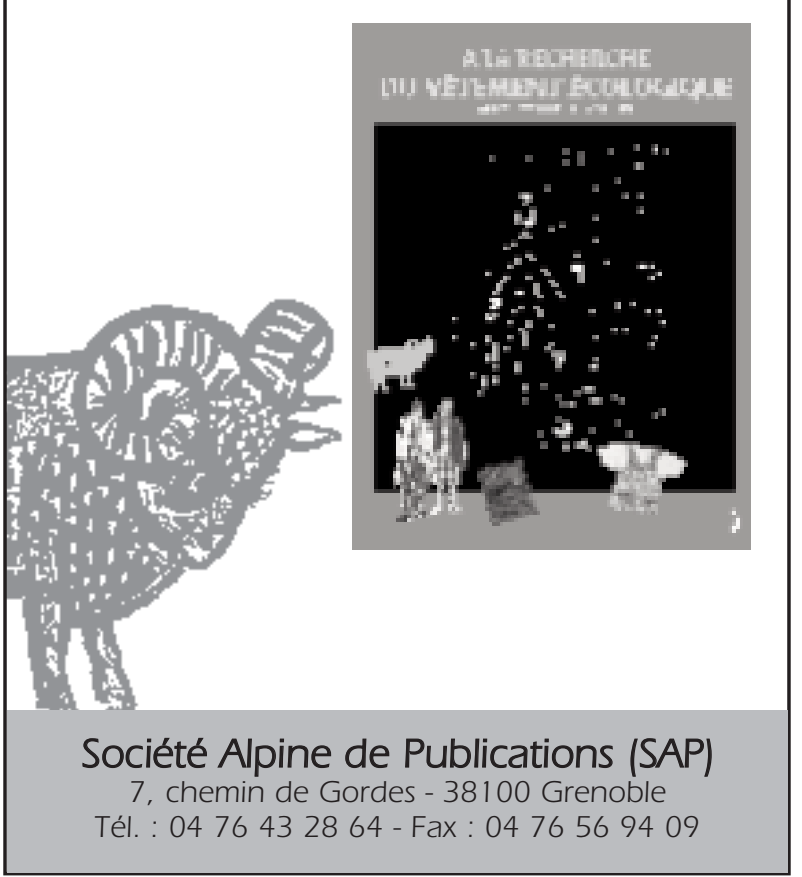

